\title{
Specific Heat Behavior in the Kondo Lattice Model With Hybridization Interaction Under Low Temperature Regimes
}

\author{
Okunzuwa I.S. \\ Arthur I. I Ejere \\ Oni-Ojo E. Amenaghawon \\ Department of Physics, Faculty of Physical Sciences, \\ University of Benin, Benin city, Nigeria \\ Aigbekaen E. Eddy \\ Department of Physics, Faculty of Natural and Applied Sciences, \\ Igbinedion University, Okada, Edo state, Nigeria
}

Doi: 10.19044/esj.2018.v14n6p405 URL:http://dx.doi.org/10.19044/esj.2018.v14n6p405

\begin{abstract}
A finite size analysis of the Kondo lattice model with hybridization interaction between localized and extended orbitals using the exact diagonalization technique has brought more insight into the specific heat behavior of the heavy fermion (HF) systems under low temperature regimes. The specific heat under the antiferromagnetic region of temperatures below $3 \mathrm{k}$ is lower than that of the ferromagnetic region. While the specific heat for temperatures above $4 \mathrm{k}$, shows a reversed trend.
\end{abstract}

Keywords: Average energy, Ferromagnetism, Ground State energy, partition function, specific heat

\section{Introduction}

The investigation of HF systems or metals with heavy electrons has developed into a new branch of metal physics. Often these systems contain $\mathrm{Ce}, \mathrm{Yb}, \mathrm{U}$ or $\mathrm{Np}$ as one of their constituents, implying that $4 \mathrm{f}$ or $5 \mathrm{f}$ electrons are involved. Characteristics examples are: $\mathrm{CeAl}_{3}, \mathrm{CeCu}_{2} \mathrm{Si}_{2}, \mathrm{CeRu}_{2} \mathrm{Si}_{2}$, $\mathrm{CeCu}_{6}, \mathrm{YbAl}_{3}, \mathrm{YbCu}_{2} \mathrm{Si}_{2}$ etc [Coqblin, 2009]. Below a characteristics temperature (usually on the order of a few Kelvin up to a few tens of Kelvin) heavy - fermion systems show Fermi-liquid behavior with large effective masses of the quasiparticles. As the temperature increases above a certain critical value, the excitations lose their HF behavior i.e. the specific heat level drops. At present there are two different physical processes which result in HF 
behavior. In both of these cases the weak interaction of f-electrons with conduction electrons constitutes the origin of the heavy quasiparticle excitations, the differences being that in one case the interactions among conduction electrons can be neglected, while in the other strong correlations between them are essential [Amadon and Hirsh, 1996]. We shall restrict ourselves to the first case which we will call standard HF behavior, because most of the systems belong to that category. We can relate the anomalous lowtemperature properties of standard HF systems to the weak hybridization of the $\mathrm{f}$ electrons with the electrons of the neighboring atoms. Because the overlap between the corresponding wave functions is so small, the strong coulomb repulsions between the f electrons suppress charge fluctuations to a large extent, thereby generating low energy excitations.

Many approximation techniques, however, were developed in the $80 \mathrm{~s}$ based on treating $1 / \mathrm{N}$ as a small parameter'. These methods were used to calculate the one electrons density of states and the dynamics susceptibility [Coqblin, 2010]. What they clearly showed was the buildup of a narrow many body resonance in the density of states at the Fermi level in the Kondo regime known as the Kondo resonance. The many body calculations giving exact results for the models or approximate ones within controlled approximations have extended the range of theoretical predictions and enabled some quantitative comparison between theory and experiment to be made [Basylko et al, 2008]. Specific heat properties of many physical quantities involve electronic excitations with energies of the order of KBT. These energies are usually of the order of or less than 10-2 ev and therefore much smaller than the Fermi energy (on the order of a few electron volt) [Desbrosavljevic and Kotliac, 1992; Hundley et al, 1994; Reich and Falicove, 1998]. This implies that the excited electrons are close to the Fermi surface which makes their effective scattering due to the interaction with other elections small. In this paper, we will systematically study the behavior of the specific heat of the Kondo lattice model (KLM) with hybridization interaction between the localized orbitals and the extended orbital under different temperature regimes. The exact diagonalization technique would be used to obtain the energy spectrum (eigenvalues) covering both the antiferromagnetic coupling $(\mathrm{J}>0)$ and ferromagnetic coupling $(\mathrm{J}<0)$ regions. There would be systematic variation of the hybridization term under various temperature regimes. The effect of the hybridization between the $\mathrm{f}-$ and the $\mathrm{c}-$ orbitals would be discussed.

\section{Methodology}

The ground state of the ID KLM is a spin liquid with new fixed points: the kondo spin liquid and the Haldane gap state. The Hamiltonian of the ID $\mathrm{KLMH}$ with hybridization interaction is written with standard notation as: 
$H=-t \sum_{t} \sum_{j=1}^{L}\left(C_{j \sigma}^{+} C_{j+\sigma}+H . C.\right)+J \sum_{j=1}^{L} S_{j c} . S_{j f}+\mathrm{V} \sum_{\sigma} \sum_{j=1}^{L}\left(C_{j \sigma}^{+} F_{j+1 \sigma}+F_{j \sigma}^{+} C_{j+1 \sigma}\right)$

Where,

$S_{j c}=\sum_{\sigma \sigma} C_{j \sigma}^{+}\left(\frac{1}{2} \sigma\right)_{\sigma \sigma} C_{j \sigma}$

$S_{j f}=\sum_{\tau \tau} F_{j \tau}^{+}\left(\frac{1}{2} \sigma\right)_{\tau \tau} F_{j \tau}$

Where $\mathrm{L}$ is the number of sites. Here we restrict ourselves to the halffilled case. We impose the periodic boundary condition and set $t=1$ as energy units. The Kondo coupling is usually antiferromagnetic $(\mathrm{J}>0)$. However, the Kondo coupling becomes ferromagnetic when there is a direct Hund coupling between $\mathrm{c}$ and $\mathrm{f}$ electrons. This Hund coupling may generate a mixing term between the $\mathrm{c}$ and f orbitals [Heershe et al, 2005;Magalhaes, et al, 2005]. The mixing (or hybridization) term is the, v. Again, it should be noted that $\mathrm{J}<0=$ JHund (Hund coupling). The ground state energy is determined using the exact diagonalization method on equation 1 and also with the aid of wolfram mathematica 9 software which is effective in diagonalizing large matrix sizes. We considered a systems of 4 electrons on 4 lattice sites and a 70 x 70 matrix size was obtained representing the 70 states in the Hilbert space.

The ground state energy $E_{\mathrm{g}}$ obtained is

$E g=\frac{1}{2}\left[J-\sqrt{J^{2}-2 J+16 t^{2}+8 v}\right]$

The specific heat of the system is determined by first calculating the partition function $\mathrm{Z}$ using

$Z=\operatorname{Tr}\langle i|\exp (-\beta H)| j\rangle$

Where $\beta=1 / k T$

Hence, $Z=2 \exp (-4 \beta v)+4 \exp (-\beta(J / 2+4 v))$

The expectation value $\langle H\rangle=E$ is defined as follows

$E=\frac{1}{Z}\langle i|H \exp (-\beta H)| j\rangle$

Equation (5) is the thermal average of the quantity H. From equation (5) we therefore obtained $\mathrm{E}$ as

$E=\frac{8 v \exp (-4 \beta v)-2 t \exp (\beta t)+4 \emptyset \exp (-\beta \emptyset)+2 t \exp (-\beta t)}{2 \exp (-4 \beta v)+4 \exp \left(-\beta\left(J /{ }^{2}+4 v\right)\right)}$ 
Where $\emptyset=J / 2+4 v$

Hence, our expression for specific heat is given by equation (7a).

$C_{v}=\frac{\partial E}{\partial T}$

Hence, $C_{v}=\frac{\Gamma(\gamma-\Omega)}{\theta}$

Where,

$$
\begin{aligned}
& \Gamma=\frac{4 v}{K T^{2}} \exp (-4 v / K T)+\frac{2 \emptyset}{K T^{2}} \exp \left(-\frac{\emptyset}{K T}\right) \\
& Y=4 v \exp (-4 v / K T)-t \exp \left(\frac{t}{K T}\right)+2 \varnothing \exp \left(-\frac{\emptyset}{K T}\right)+t \exp \left(-\frac{t}{K T}\right) \\
& \Omega=\frac{16 v^{2}}{K T^{2}} \exp (-4 v / K T)+\frac{t^{2}}{K T^{2}} \exp \left(\frac{t}{K T}\right)+\frac{2 \emptyset^{2}}{K T^{2}} \exp \left(-\frac{\emptyset}{K T}\right)+\frac{t^{2}}{K T^{2}} \exp \left(-\frac{t}{K T}\right) \\
& \theta=\exp \left(-\frac{8 v}{K T}\right)+4 \exp \left(-\frac{(4 v+\varnothing)}{K T}\right)+4 \exp \left(-\frac{2 \emptyset}{K T}\right)
\end{aligned}
$$

\section{Results And Discussion}

We show specific heats as a function of $\mathrm{J}$. Two regions are considered for $\mathrm{J}$, that is the antiferromagnetic region $(\mathrm{J}>0)$ and the ferromagnetic region $(\mathrm{J}<0)$.

In fig. $1, \mathrm{~J}$ is set to run from $\mathrm{J}=-10$ to $\mathrm{J}=9$ to enable the easy observation of the behavior of the specific heat under these two regions. This result is obtained by exact diagonalization of systems with up to 4 sites. We worked with a low temperature of $T=1.0 \mathrm{k}$. Under these conditions, the specific heat is found to be negative in nature for $J>0$. Although naturally, negative specific heat have few materials on earth expressing this rare phenomenon. It is readily observed in stars [Rennie, 2014].

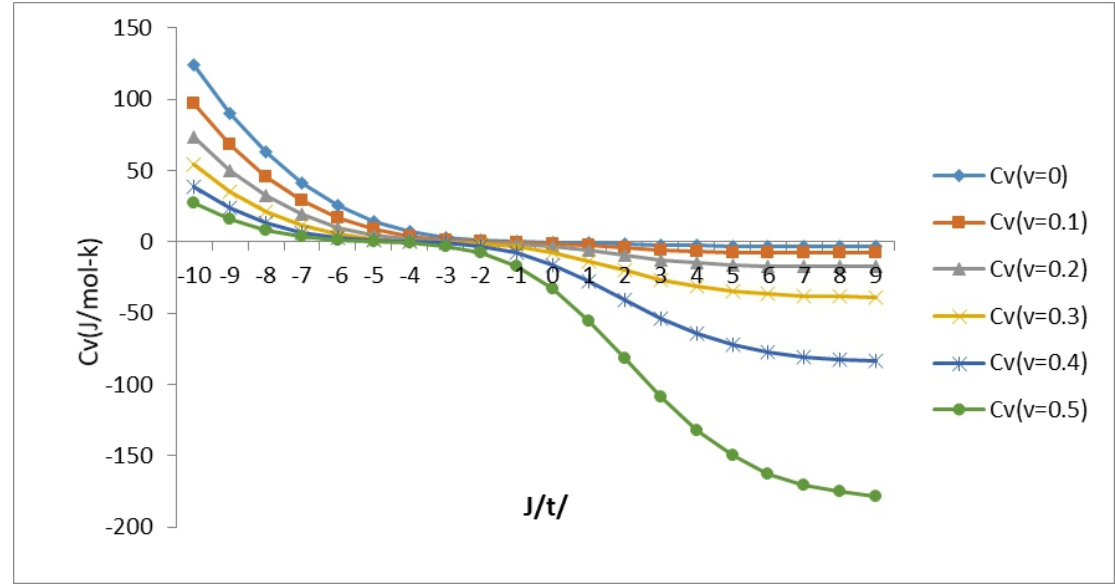

Fig 1: $\mathrm{Cv}$ vs $\mathrm{J}$ as $v$ ranges from 0.0 to 0.5 for $\mathrm{t}=1$ 
From the graph, the increase in hybridization, v from 0.1 to 0.5 pushes the specific heat into this rare natural occurrence of negative specific heat. However, we obtained positive specific heat for $\mathrm{J}<0$. Although, increase in $\mathrm{v}$ tends to reduce the specific heat at $\mathrm{T}=1.0 \mathrm{k}$.

This can be explained thus: the ferromagnetic interaction between the $\mathrm{c}$ and $\mathrm{f}$ electrons tend to produce parallel spins after interactions at the various sites and this keeps away spins according to the Pauli principles. This arrangement lowers the total energy of the system and increases the specific heat. However, hybridization between the localized and extended orbital weakens this arrangements and more localized spins become excited. This leads to a suppression of the parallel spin arrangement, and a lowering of the specific heat by extension.

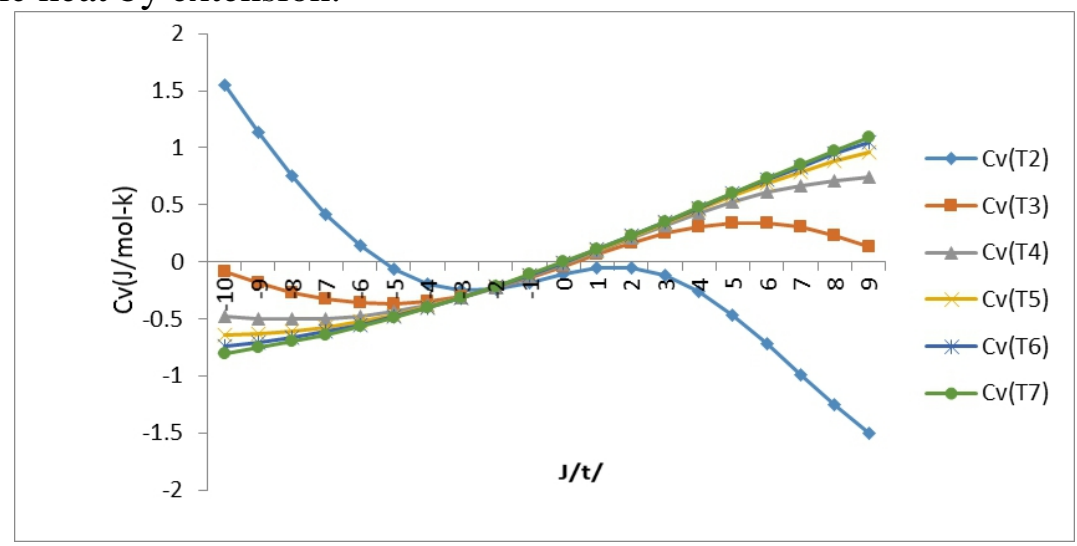

Fig 2: $\mathrm{Cv}$ vs $\mathrm{J}$ as $\mathrm{T}$ ranges from $2 \mathrm{k}$ to $7 \mathrm{k}$ for $\mathrm{t}=1$

For the higher temperature case ( $\mathrm{T}>1 \mathrm{~K})$, Fig. 2 shows functions of $\mathrm{J}<0$ and $\mathrm{J}>0$. As in the $T=1 \mathrm{k}$ case, the specific heat for $\mathrm{T}=2 \mathrm{k}$ is been weakened by $\mathrm{v}$, but the temperature range plays more role in the emergence of the properties of the system. Apart from $\mathrm{T}=2 \mathrm{k}$, that shows similar result under the ferromagnetic and antiferromagnetic regimes, higher temperatures i.e. $\mathrm{T}>3 \mathrm{k}$ recorded positive specific heat for $\mathrm{J}>0$ and negative specific heat for $\mathrm{J}<0$.

This result is due to the destruction of the Curie-Weiss condition at higher temperature leading to the suppression of the heavy fermion behavior. Under a constant hybridization of $v=0.1$, increase in temperature decouples the $\mathrm{c}$ and $\mathrm{f}$ electrons at each site under the ferromagnetic limit thereby suppressing the specific heat. While in the antiferromagnetic limits $(\mathrm{J}>0)$ the specific heat increases with temperature. 


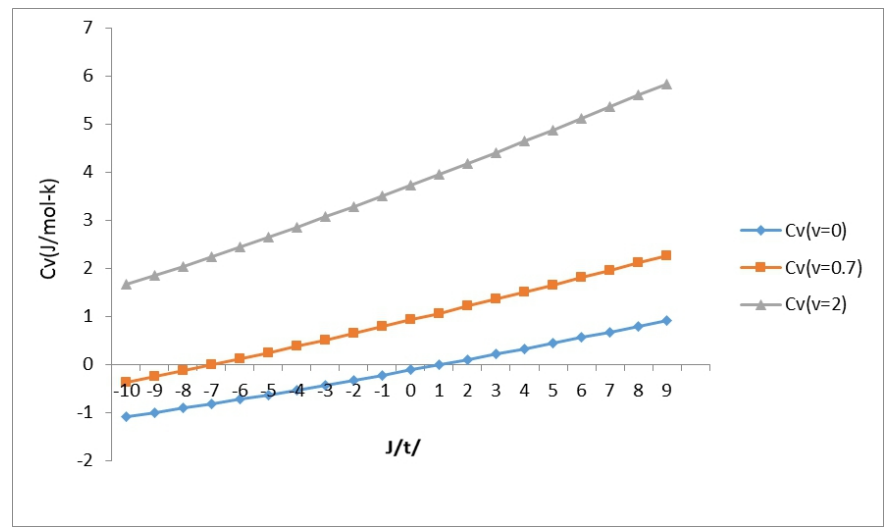

Fig 3: $\mathrm{Cv}$ vs $\mathrm{J}$ for $\mathrm{v}=0,0.7,2.0$ for $\mathrm{t}=1$

Fig. 3 shows the effects of higher temperature ( $\mathrm{T}=20 \mathrm{k}$ ), with an increase in hybridization. Apart from $\mathrm{v}=0$ and $\mathrm{v}=0.7, \mathrm{v}=2$ shows an all position specific heat with its values lower in the $\mathrm{J}<0$ limit compared to the $\mathrm{J}>0$ limit. The interplay between temperature and hybridization at higher values presents a near linear curve for the specific heat. A further increase upto $\mathrm{T}=100 \mathrm{k}$ and $\mathrm{V}=10$ leads to a stabilization of the linear increase in specific heat as seen in fig. 4.

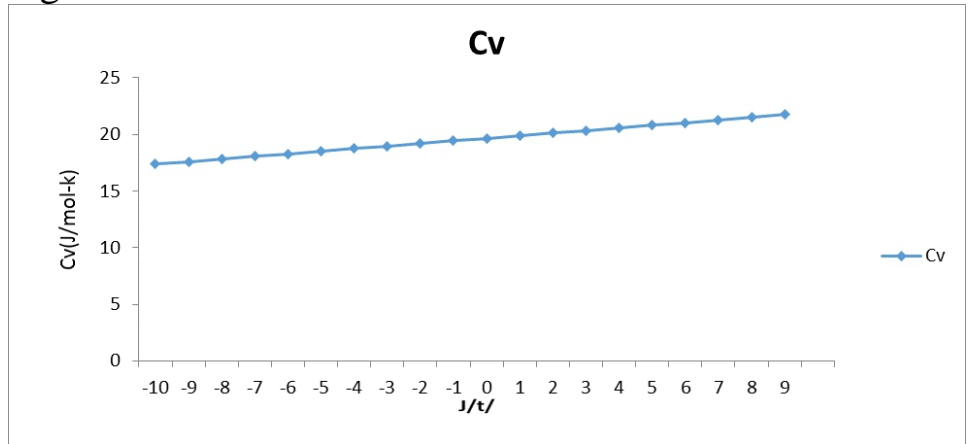

Fig 4: $\mathrm{Cv}$ vs $\mathrm{J}$ for $\mathrm{v}=10.0$ and $\mathrm{T}=100 \mathrm{k}$ for $\mathrm{t}=1$

\section{Summary And Conclusion}

In this paper, we have studied systematically the specific heat of the 1D Kondo lattice model with hybridization interaction between the $\mathrm{f}$ and $\mathrm{c}$ orbital by using the exact diagonalization for both $\mathrm{J}<0$ and $\mathrm{J}>0$. Our analysis showed the effects of temperature on the specific heat with varying hybridization values v. An increase in v generally suppresses the specific heat of the Kondo system. The ground state of the system is a singlet for $\mathrm{J}>0$ limit and the specific heat has an increasing negative value while $\mathrm{J}<0$ favors a positive specific heat under a low temperature regime of $\mathrm{T}=1 \mathrm{k}$. However, at the high temperature regime, a negative specific heat was observed for $\mathrm{J}<0$ and a positive value for specific heat was observed for $J>0$. This is due to the destruction of the Curie Weiss condition at higher temperature (Marcano et al, 
2005; Trebst et al, 2006). One of the important result of this research is the stability of the stability of specific heat at higher temperature $(T>20 \mathrm{k})$ and hybridization. Although, specific heat under $\mathrm{J}>0$ is usually higher than that of $\mathrm{J}<0$. Coqblin, (2010) used the kondo model for studying magnetism in Cerium and Uranium compounds. The introduction of the hybridization potential in the model is novel including its use in the study of specific heat. The use of the density matrix renormalization group techniques on the model is currently on going to test the results obtained here.

\section{References:}

1. Coqblin, B. (2009). Kondo Lattice and Magnetic properties in Strongly correlated electron system. Acta physica polonica A, Vol 115, 13 - 18.

2. Amadon, J.C. and Hirsh, J.E.C. (1996). Metallic ferromagnetism in a single band model: Effect of band filling and coulomb interaction. Phys. Rev. B54: 6364-6375.

3. Coqblin, B. (2010). Kondo Lattice Models and magnetism for Cerium and Uranium Systems. Acta Physica Polonica A, vol. 118, 913-918.

4. Basylko, S.A., Lundow, P.H. and Rosengren, A. (2008).The Kondo Lattice Model studied through numerical diagonalization. Phys. Rev. B78, 1-4.

5. Desbrosavljevic, V. and Kotliac G. (1992). Strong- coupling limit of the Kondo problem. Phys. Rev. B44, 7486 - 7498.

6. Hundley, M.F., Canfield, P.C., Thompson, J. D. and Fisk, Z. (1994). Subsitutional effects on the electronics transport of the kondo semiconductor Ce3Bi4 pt3. Phys. Rev. B50, 18142-18146.

7. Chen, C., Reich, A. and Falicove, L.M. (1988). Surface properties of a heavy fermion system: An exact many body solution to a periodiccluster Hubbard model. Phys. Rev. B38, 12823 - 12833.

8. Heershe, H.B., de Groot, Z., Folk, J.A., Kouwenhoven, L.P. and Van der Zant, H.J. (2005).The Kondo effect in the presence of magnetic impurities. Phys. Rev. B75, 1-5.

9. Magalhaes, S.G, Zimmer, F.M. and Coqblin,B. (2011). The van Hemmen-Kondo model for disordered Cerium systems. J. Phys:Condens. Mattter 23, 094207

10. John Rennie (2014)-Thermodynamics-Explanation for negative specific heat capacities-https://physics.stackexchange.com

11. Marcano, N. Espeso, J.I., Gomez Sal, J.C. and Rodriguez Fernandez, J. (2005). Magnetic ground state of CeNixCu1-x :A calometric Investigation. Phys. Rev. B71, 134401-134410.

12. Trebst, S., Monien, H., Grzesik, A. and Sigrist, M. (2006). Quasiparticle dynamics in the kondo lattice model at half filling. Phys. Rev. B73, 165101-165107. 